\title{
A rare case of Alport syndrome, atypical hemolytic uremic syndrome and Pauci- immune crescentic glomerulonephritis
}

\author{
Jianling Tao ${ }^{1}$, Jonathan Lieberman², Richard A. Lafayette ${ }^{1}$ and Neeraja Kambham³
}

\begin{abstract}
Background: Renal thrombotic microangiopathy (TMA) is occasionally seen in biopsies with pauci-immune necrotizing crescentic glomerulonephritis (PCGN). Recent study indicated that the complement activation is more prominent in the ANCA-negative glomerulonephritis.

Case presentation: We report a case of concurrent TMA and PCGN without ANCA positivity. Interestingly, our patient also had biopsy features supportive of Alport syndrome (AS). Genetic studies identified variants and polymorphisms in alternative complement pathway genes that confer substantial risk of developing atypical hemolytic uremic syndrome (aHUS).
\end{abstract}

Conclusions: Abnormal activation in complement pathway may represent a common pathogenic link between these three distinct entities.

Keywords: Atypical hemolytic uremic syndrome, Pauci-immune glomerulonephritis, Alternative complement pathway, Thrombotic microangiopathy

\section{Background}

Over the last several years, the causative role of the alternative complement pathway in atypical hemolytic uremic syndrome (aHUS) has been confirmed. Anti-complement therapy has been shown to be beneficial in aHUS. Pauci-immune crescentic glomerulonephritis (PCGN) lacks immune complex deposition and has not traditionally been associated with complement abnormalities. We present a patient with biopsy evidence of thrombotic microangiopathy (TMA), Alport syndrome (AS) and glomerular crescents and discuss potential pathogenic link.

\section{Case report}

\section{Clinical Presentation}

A 26-year-old woman presented with progressive shortness of breath. Her past medical history was notable for asthma diagnosed at age 3, hearing loss at age 23 (that was not fully worked up) and hypertension at age 24 .

\footnotetext{
* Correspondence: neeraja.kambham@stanford.edu

${ }^{3}$ Department of Pathology, Stanford University, Stanford, CA, USA

Full list of author information is available at the end of the article
}

Ten months prior to admission, her blood pressure was $143 / 91 \mathrm{mmHg}$, and urine dipstick detected large protein and blood, but she was lost to follow up. The family history was significant for asthma and hypertension in her mother, type II diabetes mellitus in her father and asthma in her brother; her sister was healthy.

On admission, she was afebrile, blood pressure was $191 / 125 \mathrm{mmHg}$ and the physical examination was otherwise unremarkable; fundoscopy was not performed. Urine analysis detected 25-50 non-dysmorphic RBCs/ HPF, 5-10 WBCs/HPF, $100 \mathrm{mg} / \mathrm{dl}$ protein, positive leukocyte esterase, and numerous "muddy brown casts". Relevant laboratory results were white blood cell count $7.2 \times 10^{9} / \mathrm{L}$, hemoglobin of $8.9 \mathrm{~g} / \mathrm{dL}$, platelet count $73,000 / \mu \mathrm{L}$, BUN $71 \mathrm{mg} / \mathrm{dL}$, and serum creatinine 10.11 $\mathrm{mg} / \mathrm{dL}$. Other studies include AST $34 \mathrm{u} / \mathrm{L}$, ALT $36 \mathrm{u} / \mathrm{L}$, haptoglobin $10 \mathrm{mg} / \mathrm{dl}$ (low), and LDH $2331 \mathrm{u} / \mathrm{L}$ (very high). Occasional schistocytes were seen on peripheral smear and ADAMTS13 was normal. Serological studies were normal including C3 (138), C4 (37.2), ANA (neg), anti-MPO $(<0.2)$, anti-PR3 $(<0.2)$, anti-GBM antibodies $(<0.1)$, hepatitis B, hepatitis C, HIV, and serum and urine immunoelectrophoresis. Chest $x$-ray showed

(C) The Author(s). 2018 Open Access This article is distributed under the terms of the Creative Commons Attribution 4.0 International License (http://creativecommons.org/licenses/by/4.0/), which permits unrestricted use, distribution, and 
pulmonary congestion and kidneys were echogenic on ultrasound. The patient was started on hemodialysis and a kidney biopsy was performed.

\section{Kidney Biopsy \#1}

The light microscopy sample had only 2 glomeruli and they had circumferential cellular crescents (Fig. 1a) and compressed capillary loops. Prominent tubular atrophy and interstitial fibrosis was seen with sparse interstitial inflammation and a few interstitial foam cells. The interlobular arteries and arterioles were mostly well preserved, but one arteriole had endothelial swelling, karryorrhexis and fibrin thrombus (Fig. 1b). No vasculitis was identified. Immunofluorescence revealed one glomerulus (also with crescent) and segmental granular C3 (1+; scale 0-4). No staining was seen with IgG, IgA, $\operatorname{IgM}, \mathrm{C} 1 \mathrm{q}, \mathrm{k}$ and $\lambda$. Three glomeruli, again with crescents, were available for ultrastructural examination. In addition to the extravasated fibrin and basement membrane rupture, focal endothelial cell damage was apparent with expansion of the subendothelial space by electron lucent material. Several capillary loops had basement membrane irregularities and multi-lamination of lamina densa (Fig. 1c). No electron dense deposits were identified and podocytes overlying these compressed capillary loops displayed prominent foot process effacement.

The biopsy findings were most compatible with crescentic glomerulonephritis without extrarenal manifestations. Occasional arterioles had histological features of TMA. The glomerular basement membrane changes raised concern for Alport syndrome (AS), but due to the paucity of tissue sample, type IV collagen staining could not be performed. Given the suboptimal sample and concern for systemic TMA and AS, a second biopsy was performed 3 weeks after admission.
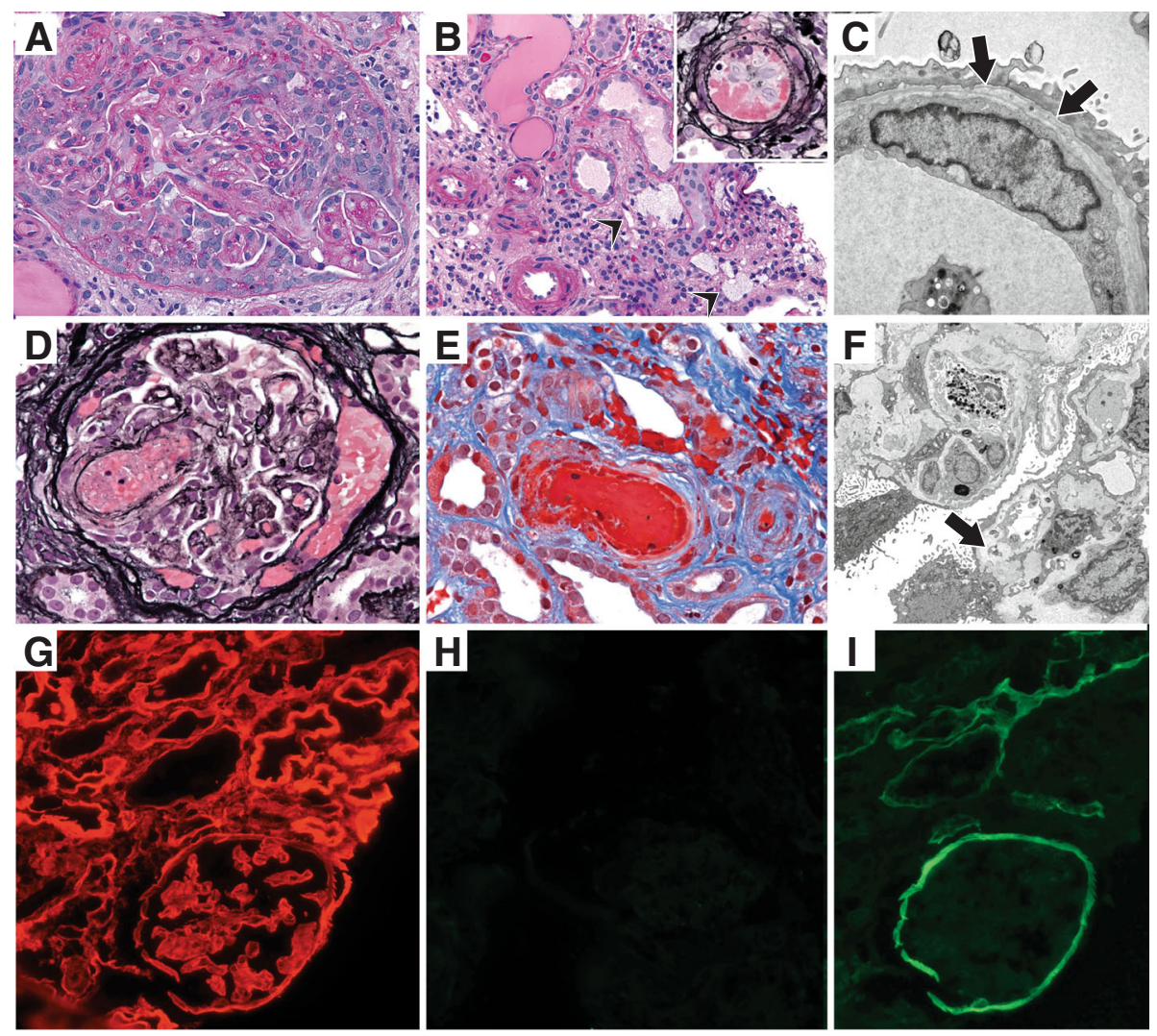

Fig. 1 Renal Biopsy Findings: a-c: Biopsy \#1. a: A circumferential cellular crescent is seen $(P A S, \times 400)$ b: There is prominent tubular atrophy and interstitial fibrosis along with interstitial foam cells (arrowheads) (H\&E, $\times 300$ ). Most arterioles are mildly thickened, but an arteriole shows intraluminal fibrin with karyorrhectic debris (inset, JMS) c: Lamina densa lamellation (arrows) is seen along the glomerular basement membrane on electron microscopy $(\times 8000)$. d-i: Biopsy \#2. d: Glomerulus with fibrin thrombus and fibrinoid necrosis near the vascular pole $(J M S \times 400)$ e: Arteriole with fibrin thrombus, karyorrhectic debris and extravasated red blood cells. No vasculitis is seen (trichrome $\times 400$ ). f: Ischemic retraction and thickening of the glomerular basement membranes on electron microscopy. Mesangiolysis and expansion of the subendothelial space is seen (arrow) (× 3000). $\mathbf{g}$ : Tissue antigen preservation documented with type IV collagen a2 staining $\mathbf{h}$ : Complete absence of a3 staining in the glomerular and tubular basement membranes i: Absent glomerular basement membrane staining with type IV collagen a5. But linear staining along the Bowman's capsule and distal tubular basement membranes is preserved 


\section{Kidney Biopsy \#2}

Eighteen glomeruli were sampled for light microscopy and three were globally sclerosed. Cellular/fibrocellular crescents were seen in 8 glomeruli with one fibrous crescent. Fibrin thrombi were identified in two glomeruli (Fig. 1d). Several other glomeruli had ischemic changes and the tubular atrophy and interstitial fibrosis involved over $80 \%$ of the cortex. Clusters of interstitial foam cells were seen throughout the cortex. Several cross sections of interlobular arteries and arterioles had intimal edema, fibrinoid necrosis, and concentric thickening of the wall; no vasculitis was identified (Fig. 1e). Immunofluorescence was negative. On ultrastructural examination of 3 ischemic glomeruli, extensive endothelial cell injury was seen with subendothelial electron lucent material; prominent mesangiolysis was also present (Fig. 1f). Lamina densa lamellation was again seen. Additional immunofluorescence was performed for type IV collagen $\alpha 2$, $\alpha 3$ and $\alpha 5$ chains (Fig. 1g-i). Alpha2 staining was normal, indicative of good antigen preservation. However, $\alpha 3$ was completely absent in both glomeruli and tubules, and $\alpha 5$ lacked staining along the glomerular basement membranes but had intact staining along Bowman's capsules and distal tubular basement membranes.

\section{Immunohistochemical Studies}

Both kidney biopsies were stained for C4d (rabbit polyclonal, cat. no. 04-B1-RC4D, Biomedica, Austria, 1/20 dilution) and C5b-9 (mouse monoclonal, cat.no. M0777, DAKO, Denmark, 1/10 dilution). Intimal and medial staining was seen with both C4d and C5b-9 in arterioles affected by thrombotic microangiopathy. Glomerular staining was seen in crescents and sclerosed segments, albeit more strongly with C5b-9 (Fig. 2). In addition, C5b-9 staining was seen in patchy peritubular capillaries and interstitium.

\section{Diagnosis and follow-up}

Both kidney biopsies demonstrate crescentic glomerulonephritis, with increased chronicity on the second biopsy. In addition, there was acute and subacute TMA, better visualized on second biopsy. A sequencing of 12 aHUS-related genes was performed using a database of over 400 aHUS-associated mutations, disease-associated polymorphisms, benign polymorphisms and other known variants of undetermined significance (Machoan Diagnostics, Oakland, CA). Our patient was negative for the large CFHR1-CFHR3 homozygous deletion associated with $\mathrm{CFH}$ auto-antibodies. However, she had a heterozygous missense variant (c.1246 A $>$ C, p.lle416Leu) in exon 11 of $C F I$, reported in aHUS [1, 2]. A heterozygous missense variant (c.136C $>$ T, p. Pro46Ser) in exon 2 of CFHR5, reported in dense deposit disease was also noted [3]. A heterozygous polymorphism (IVS9-78 G >
A) within an intron in $M C P / C D 46$ was present and there were also 5 SNPs near and within $M C P / C D 46$ that comprised a haplotype, also associated with increased risk for aHUS [4].

The clinical, laboratory and genetic findings indicate a diagnosis of aHUS coexistent with glomerular crescents seen on biopsy. The interstitial foam cells on light microscopy (in the absence of chronic nephrotic proteinuria), basement membrane abnormalities on electron microscopy and abnormal immunofluorescence for $\alpha 3$ and $\alpha 5$ chains of type IV collagen support a diagnosis of AS, that likely contributed to the underlying chronic kidney disease. The immunofluorescence pattern of $\alpha 3$ and $\alpha 5$ staining suggests a $\alpha 3$ gene mutation with autosomal recessive inheritance. A genetic analysis for AS was suggested.

The patient was treated on admission with pulse methylprednisolone followed by oral prednisone. Daily plasmapheresis was initiated, but was discontinued after a few days once the ADAMTS13 levels were found to be normal. Cytoxan was administrated after the first biopsy results. Eculizumab therapy was initiated after the second biopsy and she continued to receive it every 2 weeks at her 8-month follow up. Her kidney function remains poor requiring maintenance hemodialysis.

\section{Discussion and conclusions}

Atypical hemolytic uremic syndrome (aHUS) is relatively rare, and studies over the last decade have vastly improved our understanding of the disease $[5,6]$. In approximately $60 \%$ of cases, aHUS is linked to mutations in complement genes encoding both regulatory and activation proteins involved in the complement pathway. Disease results from either a loss-of-function mutation in a regulatory gene (eg. $C F H, C F I$, or CD46) or a gain-of-function mutation in an effector gene (eg. $C F B$ or $C 3)$, resulting in alternative complement pathway dysregulation on cell surfaces. $C F H$ mutation-associated aHUS, the most common form, comprises $20-30 \%$ of inherited disease [7]. In a subset of patients, similar to ours, combinations of gene abnormalities result in aHUS [5]. The disease penetrance is variable and several environmental triggers such as infections, pregnancy, respiratory illness and drugs likely precipitate overt aHUS. The precipitating factor in our patient is unclear. Only a third of aHUS patients have low serum C3 levels, usually in association with complement gene mutations. The biopsy findings of aHUS are those of TMA involving glomeruli and/or small arteries and arterioles.

The prevalence of alternative complement pathway dysregulation in disease appears to be greater than previously appreciated. Timmermans et al [8] examined complement abnormalities in nine patients with malignant hypertension-associated TMA, and found mutations in 


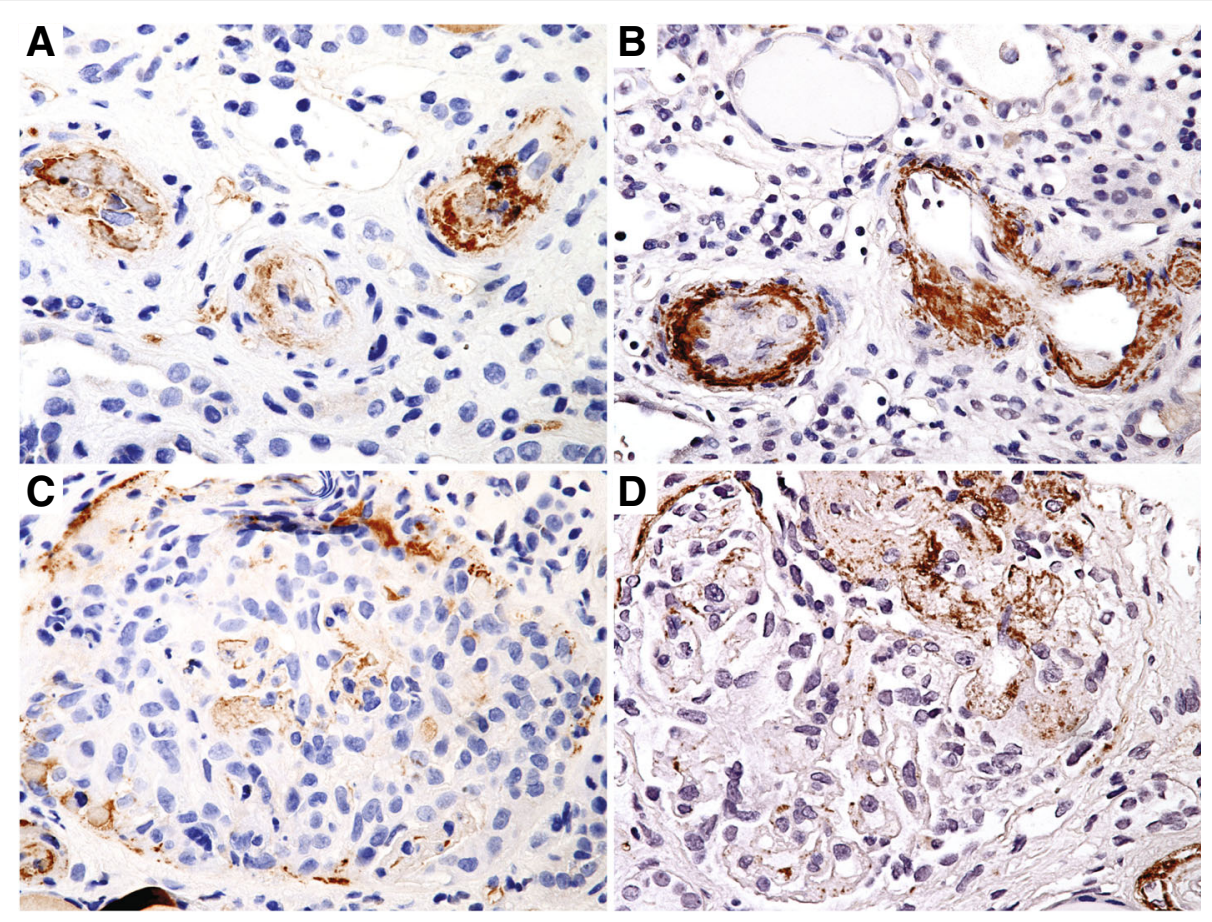

Fig. 2 Immunohistochemical evidence of complement pathway activation. a: Arteriolar C4d staining within intimal and adjacent media in vessels affected by acute thrombotic microangiopathy (biopsy \#1) (× 400) b: Arteriolar C5b-9 (membrane attack complex) staining in affected vessels (biopsy \#1) (× 400) c: Weak glomerular staining with C4d in a crescent $(\times 400)$ d: Glomerular C5b-9 staining predominantly within a cellular crescent (biopsy \#2) $(\times 400)$

C3, CFI, CD46, or CFH genes in six. Complement activation in these patients was evidenced by elevated serum soluble C5b-9 levels and renal deposits of $\mathrm{C} 3 \mathrm{c}$ and C5b-9. Co-localization of C4d deposits in vasculature and glomerular capillary walls indicates activation of classical and/or lectin pathway as well. Our patient did show systemic evidence of TMA (akin to malignant hypertension) with circulating schistocytes and elevated LDH levels. Chua et al [9] evaluated for C4d and C5b-9 deposits in 42 renal biopsies (both native and transplant) with TMA related to heterogeneous underlying diseases. C4d and C5b-9 deposits were quite common, although the pattern of staining was variable based on etiology. Our patient had deposits of both C4d and C5b-9, providing immunohistochemical support for complement pathway activation. Continuous alternative complement pathway abnormalities in aHUS patients result in endothelial dysfunction and formation of microvascular thrombi. Eculizumab is a humanized monoclonal IgG antibody that binds to complement protein $\mathrm{C} 5$, preventing cleavage into $\mathrm{C} 5 \mathrm{a}$ and $\mathrm{C} 5 \mathrm{~b}$. Blocking the formation of $\mathrm{C} 5 \mathrm{~b}$ interrupts this complement cascade and membrane attack complex (C5b-9)-mediated injury. Several studies have shown the safety and efficacy of eculizumab therapy in patients with aHUS [10].

Alport syndrome (AS), which usually manifests as a triad of hematuria, senisorineural hearing loss and ocular symptoms, is caused by the disruption of type IV collagen, a key component of the glomerular basement membrane. Specifically, mutations in the COL4A5, COL4A3 or COL4A4 genes, which encode the $\alpha 3, \alpha 4$, and $\alpha 5$ chains of collagen type IV respectively, cause the disease [11]. Over 500 mutations have been described, which result in disease with either X-linked (mutations in COL4A5; 85\% of patients) or autosomal recessive inheritance (mutations in COL4A3 and COL4A4; $15 \%$ of patients) [12]. An autosomal dominant inheritance pattern has been reported in a minority of patients $[12,13]$.

A diagnosis of AS is generally determined by the family history, presence of visual and/or hearing dysfunction, and electron microscopy findings on kidney biopsy. The glomerular basement membrane changes are typically characterized by diffuse thickening and splitting of the lamina densa. Immunofluorescence in our patient revealed total lack of $\alpha 3$ and loss of $\alpha 5$ staining in glomerular basement membranes but preserved a5 staining in Bowman capsule and distal tubular basement membranes. Mutations in the COL4A3 (or COL4A4) gene results in the defective triple helix with demonstrable $\alpha 5$ protein loss only in those basement membranes (glomerulus) in which all three proteins are associated. [14] Based on these findings, we suspect that our patient has autosomal recessive AS. 
Crescents are rarely reported in aHUS [15], but tend to be small and barely meet the criterion for a crescent [16]. It is also possible that the crescents in our patient are related to AS [17]. In reviewing 665 human Alport nephropathy biopsies, Ryu et al. [18] found glomerular crescents in $0.4 \%$ of biopsies, suggesting an extremely rare occurrence. In each of those biopsies, only $3-11 \%$ of glomeruli sampled had crescents. It has been proposed that plasma leakage due to glomerular vascular injury and glomerular basement membrane breaks might trigger parietal epithelial cell proliferation and crescent formation. Based on studies in Col4A3 -deficient mice, crescent formation occurs more frequently in late stage Alport glomerulopathy [18].

The presence of extensive crescents seen in our patient also suggests co-existent ANCA negative (antineutrophil cytoplasmic antibody anti-PR3 and anti-MPO) pauci-immune crescentic glomerulonephritis (PCGN). Review of the literature demonstrates a growing appreciation for the role of complement in PCGN $[19,20]$. Complement plays a key role in murine ANCA-associated PCGN induced by injection of anti-MPO antibodies [21]. The alternative complement pathway has been shown to be critical in the pathogenesis as mice deficient in factor B or C3 fail to develop PCGN [21]. Anaphylatoxin C5a and the neutrophil C5a receptor mediate an amplification loop for ANCA-induced neutrophil activation that triggers the complement cascade in the serum. C5a, a potent chemoattractant for neutrophils, monocytes and macrophages, also increases the membrane expression of ANCA on neutrophils, leading to reactive oxygen species generation and necrotizing vasculitis [22].

Despite the general impression, a high percentage of patients with ANCA-associated PCGN have immunofluorescence and ultrastructural evidence of immune deposits. These immunoglobulin and complement deposits are typically of low intensity [23]. Recently, Chen et al. examined 112 biopsies from patients from ANCA-associated PCGN [24]. In one third of the patients, glomerular $\mathrm{C} 3 \mathrm{c}$ deposits were detected and this feature was associated with greater proteinuria and worse renal function at presentation. Other components of alternative complement pathway activation such as factor $\mathrm{Bb}, \mathrm{C} 3 \mathrm{~d}$ and properdin deposits were also demonstrable in the kidney biopsies with ANCA-associated vasculitis [25-27] .The presence of C3d and properdin deposits correlated with more severe crescentic disease [27]. Other investigators have sought evidence of alternative pathway activation and complement consumption in serum and urine. Low serum C3 level at diagnosis of renal ANCA vasculitis is associated with lower renal function at presentation and worse renal and patient survivals [28]. Circulating serum factor Bb is elevated in active ANCA vasculitis and may in fact be a biomarker for disease activity [29]. Alternative complement pathway activation products $\mathrm{Bb}, \mathrm{C} 3 \mathrm{a}$, and $\mathrm{C} 5 \mathrm{a}$ and common terminal pathway product soluble C5b-9 were all found to be significantly elevated in the urine of patients with active compared to remission stage of ANCA PCGN [25]. There may also be activation of classic and lectin complement pathways, but the overall evidence suggests that they do not play a critical pathogenic role $[25,27,30]$.

Given the involvement of alternative complement pathway in both ANCA disease and aHUS, it seems quite plausible that these diseases occur concurrently, as has been rarely reported [31]. Manenti et al. studied 46 patients with ANCA vasculitis, and eight of 30 patients who underwent biopsy (27\%) had histologic signs of TMA [32]. This subgroup with histological evidence of TMA had a dramatically worse death-censored renal survival than patients without TMA. Interestingly, serum C3 levels were low in more than a third of these patients with ANCA vasculitis. Identifying a pathogenic role for complement in PCGN disease has significant therapeutic implications. Initiation of anti-complement therapy (eculizumab, anti-C5) immediately after a diagnosis of aHUS is associated with better renal outcome [33]. There can be a potential beneficial role of anti-complement therapy in PCGN disease as well [34, 35]. Recently, Sathe et al. [36] reported a pediatric patient with biopsy proven ANCA disease who developed aHUS 6 months later, as evidenced by low serum $\mathrm{C} 3$ and circulating anti-factor $\mathrm{H}$ antibodies. No genetic studies were performed. Complete renal recovery was achieved following treatment with intravenous rituximab, steroids, cyclophosphamide, and plasmapheresis.

Overall, it seems that TMA in ANCA disease may be under recognized both clinically and on biopsy. The identification of at risk genetic variants and polymorphisms in complement genes in our patient with both PCGN and TMA lends further support for shared pathogenic pathway in these two histologically distinct entities. Ours is a rather unusual case with a third diagnosis of AS. Glomerular crescents can rarely be seen in AS, and there are rare reports of TMA in an AS patient $[37,38])$. A 16 year old patient presented with nephrotic syndrome and the biopsy revealed chronic TMA and a diagnosis of X-linked AS was subsequently made based on whole-exome sequencing. So, while a PCGN superimposed on TMA is a distinct possibility, crescent formation may have been triggered or accentuated by both glomerular injury caused by aHUS and the compromised basement membrane integrity due to AS.

In conclusion, we report a case of AS patient with concurrent aHUS and glomerular crescents. Genetic analysis revealed at risk variants and polymorphisms in alternative complement pathway genes. We hypothesize 
that this genetic predisposition in this unfortunate patient led to precipitation of aHUS and potentially PCGN in the context of an unidentified environmental trigger.

\section{Abbreviations}

aHUS: Atypical hemolytic uremic syndrome; AS: Alport syndrome;

PCGN: Pauci-immune necrotizing crescentic glomerulonephritis; TMA: Renal thrombotic microangiopathy

\section{Acknowledgements}

None.

\section{Funding}

None.

\section{Availability of data and materials}

Not applicable as it is a case report and there is no additional data

\section{Authors contributions}

JT reviewed the literature, performed additional immunohistochemical stains and wrote the first draft of the manuscript. JB carried out the molecular genetic studies and participated in the drafting of the manuscript. RAF participated in the design of the study and provided critical feedback. NK conceived of the study, and participated in its design and coordination. All authors read and approved the final manuscript.

\section{Ethics approval and consent to participate}

Obtained as per Stanford University IRB regulations.

\section{Consent for publication}

Written informed consent was obtained from the patients for publication of this Case report and any accompanying images. A copy of the written consent is available for review by the Editor of this journal.

\section{Competing interests}

The results presented in this paper have not been published previously in whole or part. The authors confirm that they have nothing to declare. The authors declare that they have no competing interests.

\section{Publisher's Note}

Springer Nature remains neutral with regard to jurisdictional claims in published maps and institutional affiliations.

\section{Author details}

'Department of Medicine, Division of Nephrology, Stanford University, Stanford, USA. ${ }^{2}$ Department of Nephrology, Kaiser Permanente, San Francisco, CA, USA. ${ }^{3}$ Department of Pathology, Stanford University, Stanford, CA, USA.

Received: 8 June 2018 Accepted: 3 December 2018

Published online: 12 December 2018

\section{References}

1. Maga TK, Nishimura CJ, Weaver AE, Frees KL, Smith RJ. Mutations in alternative pathway complement proteins in American patients with atypical hemolytic uremic syndrome. Hum Mutat. 2010;31(6):E1445-60.

2. Bresin E, Rurali E, Caprioli J, Sanchez-Corral P, Fremeaux-Bacchi V, Rodriguez de Cordoba S, Pinto S, Goodship TH, Alberti M, Ribes D, et al. Combined complement gene mutations in atypical hemolytic uremic syndrome influence clinical phenotype. J Am Soc Nephrol. 2013;24(3):475-86.

3. Abrera-Abeleda MA, Nishimura C, Smith JL, Sethi S, McRae JL, Murphy BF, Silvestri G, Skerka C, Jozsi M, Zipfel PF, et al. Variations in the complement regulatory genes factor $\mathrm{H}$ (CFH) and factor $\mathrm{H}$ related 5 (CFHR5) are associated with membranoproliferative glomerulonephritis type II (dense deposit disease). J Med Genet. 2006;43(7):582-9.

4. Esparza-Gordillo J, Goicoechea de Jorge E, Buil A, Carreras Berges L, LopezTrascasa M, Sanchez-Corral P, Rodriguez de Cordoba S. Predisposition to atypical hemolytic uremic syndrome involves the concurrence of different susceptibility alleles in the regulators of complement activation gene cluster in 1q32. Hum Mol Genet. 2005;14(5):703-12.
5. Fremeaux-Bacchi V, Fakhouri F, Garnier A, Bienaime F, Dragon-Durey MA, Ngo S, Moulin B, Servais A, Provot F, Rostaing L, et al. Genetics and outcome of atypical hemolytic uremic syndrome: a nationwide French series comparing children and adults. Clin J Am Soc Nephrol. 2013;8(4):554-62.

6. Nester CM, Barbour T, de Cordoba SR, Dragon-Durey MA, Fremeaux-Bacchi V, Goodship TH, Kavanagh D, Noris M, Pickering M, Sanchez-Corral P, et al. Atypical aHUS: state of the art. Mol Immunol. 2015;67(1):31-42.

7. Berger BE. The alternative pathway of complement and the evolving clinical-pathophysiological Spectrum of atypical hemolytic uremic syndrome. Am J Med Sci. 2016;352(2):177-90.

8. Timmermans S, Abdul-Hamid MA, Vanderlocht J, Damoiseaux J, Reutelingsperger $C P$, van Paassen $P$, Limburg Renal R. Patients with hypertension-associated thrombotic microangiopathy may present with complement abnormalities. Kidney Int. 2017;91(6):1420-5.

9. Chua JS, Baelde HJ, Zandbergen M, Wilhelmus S, van Es LA, de Fijter JW, Bruijn JA, Bajema IM, Cohen D. Complement factor C4d is a common denominator in thrombotic Microangiopathy. J Am Soc Nephrol. 2015;26(9):2239-47.

10. Raina R, Krishnappa V, Blaha T, Kann T, Hein W, Burke L, Bagga A. Atypical hemolytic-uremic syndrome: an update on pathophysiology, diagnosis, and treatment. Ther Apher Dial. 2018. [Epub ahead of print].

11. Savige J, Gregory M, Gross O, Kashtan C, Ding J, Flinter F. Expert guidelines for the management of Alport syndrome and thin basement membrane nephropathy. J Am Soc Nephrol. 2013;24(3):364-75.

12. Kruegel J, Rubel $\mathrm{D}, \mathrm{Gross} \mathrm{O}$. Alport syndrome--insights from basic and clinical research. Nat Rev Nephrol. 2013;9(3):170-8.

13. Heidet $L$, Gubler MC. The renal lesions of Alport syndrome. J Am Soc Nephrol. 2009;20(6):1210-5.

14. Gubler MC, Knebelmann B, Beziau A, Broyer M, Pirson Y, Haddoum F, Kleppel MM, Antignac C. Autosomal recessive Alport syndrome: immunohistochemical study of type IV collagen chain distribution. Kidney Int. 1995:47(4):1142-7.

15. Zoltan G, Laszik NK, Fred G. Silva: Thrombotic Microangiopathies. In: Hepinstall's Pathology of the Kidney. Volume 2, edn. Edited by J Charlesjennette JLO, Fred G. Silva, Vivette D. D'Agati. Philadelphia: Wolters Kluwer; 2015: 739-814.

16. Taylor CM, Chua C, Howie AJ, Risdon RA. Clinico-pathological findings in diarrhoea-negative haemolytic uraemic syndrome. Pediatr Nephrol. 2004;19(4):419-25.

17. Gubler M, Levy M, Broyer M, Naizot C, Gonzales G, Perrin D, Habib R. Alport's syndrome. A report of 58 cases and a review of the literature. Am J Med. 1981;70(3):493-505

18. Ryu M, Migliorini A, Miosge N, Gross O, Shankland S, Brinkkoetter PT, Hagmann H, Romagnani P, Liapis H, Anders HJ. Plasma leakage through glomerular basement membrane ruptures triggers the proliferation of parietal epithelial cells and crescent formation in non-inflammatory glomerular injury. J Pathol. 2012;228(4):482-94.

19. Kallenberg CG, Heeringa P. Complement system activation in ANCA vasculitis: a translational success story? Mol Immunol. 2015:68(1):53-6.

20. Sethi S, Zand L, De Vriese AS, Specks U, Vrana JA, Kanwar S, Kurtin P, Theis JD, Angioi $A$, Cornell $L$, et al. Complement activation in pauci-immune necrotizing and crescentic glomerulonephritis: results of a proteomic analysis. Nephrol Dial Transplant. 2017:32(suppl_1):i139-45.

21. Xiao H, Schreiber A, Heeringa P, Falk RJ, Jennette JC. Alternative complement pathway in the pathogenesis of disease mediated by antineutrophil cytoplasmic autoantibodies. Am J Pathol. 2007;170(1):52-64.

22. Schreiber A, Xiao H, Jennette JC, Schneider W, Luft FC, Kettritz R. C5a receptor mediates neutrophil activation and ANCA-induced glomerulonephritis. J Am Soc Nephrol. 2009;20(2):289-98.

23. Haas M, Eustace JA. Immune complex deposits in ANCA-associated crescentic glomerulonephritis: a study of 126 cases. Kidney Int. 2004;65(6):2145-52.

24. Chen M, Xing GQ, Yu F, Liu G, Zhao MH. Complement deposition in renal histopathology of patients with ANCA-associated pauci-immune glomerulonephritis. Nephrol Dial Transplant. 2009:24(4):1247-52.

25. Gou SJ, Yuan J, Wang C, Zhao MH, Chen M. Alternative complement pathway activation products in urine and kidneys of patients with ANCAassociated GN. Clin J Am Soc Nephrol. 2013;8(11):1884-91.

26. Xing GQ, Chen M, Liu G, Heeringa P, Zhang JJ, Zheng X, E J, Kallenberg CG, Zhao MH. Complement activation is involved in renal damage in human antineutrophil cytoplasmic autoantibody associated pauci-immune vasculitis. J Clin Immunol. 2009;29(3):282-91. 
27. Hilhorst $M$, van Paassen $P$, van Rie $H$, Bijnens $N$, Heerings-Rewinkel $P$, van Breda Vriesman P, Cohen Tervaert JW, Limburg Renal R. Complement in ANCA-associated glomerulonephritis. Nephrol Dial Transplant. 2015;32(8): 1302-1313.

28. Augusto JF, Langs V, Demiselle J, Lavigne C, Brilland B, Duveau A, Poli C, Chevailler A, Croue A, Tollis F, et al. Low serum complement C3 levels at diagnosis of Renal ANCA-associated Vasculitis is associated with poor prognosis. PLoS One. 2016;11(7):e0158871.

29. Gou SJ, Yuan J, Chen M, Yu F, Zhao MH. Circulating complement activation in patients with anti-neutrophil cytoplasmic antibody-associated vasculitis. Kidney Int. 2013;83(1):129-37.

30. Xing GQ, Chen M, Liu G, Zheng X, E J, Zhao MH. Differential deposition of C4d and MBL in glomeruli of patients with ANCA-negative pauci-immune crescentic glomerulonephritis. J Clin Immunol. 2010;30(1):144-56.

31. Manenti L, Gnappi E, Vaglio A, Allegri L, Noris M, Bresin E, Pilato FP, Valoti E, Pasquali S, Buzio C. Atypical haemolytic uraemic syndrome with underlying glomerulopathies. A case series and a review of the literature. Nephrol Dial Transplant. 2013;28(9):2246-59.

32. Manenti L, Vaglio A, Gnappi E, Maggiore U, Allegri L, Allinovi M, Urban ML, Delsante M, Galetti M, Nicastro M, et al. Association of serum C3 concentration and histologic signs of thrombotic Microangiopathy with outcomes among patients with ANCA-associated Renal Vasculitis. Clin J Am Soc Nephrol. 2015;10(12):2143-51.

33. Legendre CM, Licht $C$, Muus $P$, Greenbaum LA, Babu S, Bedrosian $C$ Bingham C, Cohen DJ, Delmas Y, Douglas K, et al. Terminal complement inhibitor eculizumab in atypical hemolytic-uremic syndrome. N Engl J Med. 2013;368(23):2169-81.

34. Jayne DR, Bruchfeld AN, Harper L, Schaier M, Venning MC, Hamilton P, Burst V, Grundmann F, Jadoul M, Szombati I, et al. Randomized trial of C5a receptor inhibitor Avacopan in ANCA-associated Vasculitis. J Am Soc Nephrol. 2017;28(9):2756-67.

35. Manenti L, Urban ML, Maritati F, Galetti M, Vaglio A. Complement blockade in ANCA-associated vasculitis: an index case, current concepts and future perspectives. Intern Emerg Med. 2017;12(6):727-31.

36. Sathe KP, Mehta KP. Coexistence of atypical hemolytic uremic syndrome with membranoproliferative glomerulonephritis and antineutrophil cytoplasmic antibodies-associated vasculitis. Saudi J Kidney Dis Transpl. 2016;27(4):800-4.

37. Wuttke M, Seidl M, Malinoc A, Prischl FC, Kuehn EW, Walz G, Kottgen A. A COL4A5 mutation with glomerular disease and signs of chronic thrombotic microangiopathy. Clin Kidney J. 2015;8(6):690-4.

38. Sanchez-Nino MD, Ortiz A. Thrombotic microangiopathy: expanding genetic, clinical and therapeutic spectra and the need for worldwide implementation of recent advances. Clin Kidney J. 2015:8(6):686-9.

Ready to submit your research? Choose BMC and benefit from:

- fast, convenient online submission

- thorough peer review by experienced researchers in your field

- rapid publication on acceptance

- support for research data, including large and complex data types

- gold Open Access which fosters wider collaboration and increased citations

- maximum visibility for your research: over $100 \mathrm{M}$ website views per year

At BMC, research is always in progress.

Learn more biomedcentral.com/submissions 